\title{
Estado nutricional y uso de la tarjeta de alimentación JUNAEB en estudiantes de una universidad chilena
}

\author{
Nutritional status and use of JUNAEB card \\ in students of a Chilean university
}

\begin{abstract}
Introduction: The usual Chilean diet is characterized by a high energy intake from saturated fat, cholesterol, sugar and sodium in foods like fried foods, baked goods and pastries, meats, sweets and sugary drinks; and the lack of physical activity has contributed to the deterioration of the nutritional status of young adult population, as shown in the National Health Survey of 2010 which found in 15-24 years old persons a prevalence of $10.9 \%$ for obesity, $13.2 \%$ of high cholesterol and $79.3 \%$ of sedentary lifestyle. Objective: To relate the use of JUNAEB card with the nutritional status of students of the Santo Tomás University in Valdivia during the second half of 2014. Methodology: Cross sectional study involving 60 students to whom the "JUNATEST" survey was applied to assess the quality of food they purchased using the JUNAEB card and their nutritional status. Statistical analysis: STATA 12.0 and Shapiro Wilk test, Bartlett, ANOVA and Pearson correlation was used. To calculate the sample size a power of $80 \%$, confidence level of $95 \%$, an error of $5 \%$ and $p$ value $<0.05$ was used.
\end{abstract}

Key words: JUNAEB card, healthy eating, university students, nutritional status, survey JUNATEST.

\section{INTRODUCIÓN}

En nuestro país ha existido durante las últimas décadas una evolución, caracterizada por el aumento de las enfermedades crónicas no transmisibles (ECNT) y enfermedades cardiovasculares que se relacionan con estilos de vida no saludables $(1,2)$. La dieta habitual de nuestro país basada en la dieta occidental se caracteriza por un elevado aporte calórico proveniente de grasas saturadas, trans-ácidos, colesterol, azúcares simples y sodio. Otra característica importante de la alimentación en Chile es un bajo aporte de ácidos grasos omega 3, fibra, antioxidantes y minerales como calcio, hierro y zinc (3)

El deficiente estado nutricional de la población adulto joven de nuestro país se reflejó en la Encuesta Nacional de Salud 2010 que incluyó 812 jóvenes; 364 hombres y 448 mujeres entre 15 a 24 años, determinándose una prevalencia de obesidad de 10,9\% y 79,3\% de sedentarismo (1). En los resultados destaca que sólo 13\% de los encuestados cubre las recomendaciones de consumo de frutas y verduras propuestas por el Ministerio de Salud (1).

El Ministerio de Educación de Chile otorga una beca de
María José Levío A., Marion Thamara Guerrero W.

Universidad Santo Tomas Sede Valdivia,
Carrera Nutrición y Dietética
Universidad Santo Tomas, Sede Valdivia, Valdivia, Chile.
Dirigir la correspondencia a:
Profesora
Marion Thamara Guerrero Wyss
Carrera de Nutrición U. Santo Tomas, Sede Valdivia, Valdivia Chile

Este trabajo fue recibido el 14 de Abril de 2015 y aceptado para ser publicado el 31 de Julio de 2015.

alimentación para la educación superior (Beca BAES), cuyo objetivo es apoyar la permanencia y egreso de la educación superior a estudiantes vulnerables. Consiste en un subsidio de alimentación (\$32.000 mensual) entregado a través de una tarjeta electrónica de canje, que puede ser utilizada en una red de locales especializados en la venta de productos alimenticios y supermercados (4).

El objetivo de este estudio fue relacionar el uso de tarjeta JUNAEB con el estado nutricional de estudiantes universitarios de la universidad Santo Tomás, sede Valdivia durante el segundo semestre del año 2014.

\section{SUJETOS Y MÉTODO}

Para el cálculo del tamaño muestral; se consideró la estimación para una propoción del total de 289 estudiantes universitarios entre 18 y 30 años, alumnos regulares y además beneficiados con la beca de alimentación JUNAEB de las carreras de Psicología, Derecho, Enfermería y Kinesiología de la Universidad Santo Tomás, sede Valdivia durante el año 2014. Con un nivel de confianza del $95 \%$, error de $5 \%$ y un poder de $80 \%$, resultando una muestra final de 60 estudiantes es- 
tratificada por carrera, de los cuales 7 correspondieron a la carrera de Derecho, 29 a enfermería, 18 a kinesiología y 6 a la carrera de psicología.

La investigación consideró la aprobación del Comité de Ética de la Universidad Santo Tomás antes del inicio de la recolección de datos.
Los participantes fueron previamente notificados mediante un consentimiento informado; en este documento se detallaron las mediciones antropométricas y la encuesta alimentaria "JUNATEST" (Anexo 1) mediante la cual se evaluó el uso de la tarjeta JUNAEB. De acuerdo a los resultados obtenidos en ésta, se clasificó el uso de la tarjeta en cada

Anexo 1. Encuesta JUNATEST.

\section{ANEXOS}

Nombre Completo:

ID:

Edad:

Carrera:

1. Del total del dinero mensual de la tarjeta de alimentación, ¿cuánto utiliza en supermercados?

a) No la utilizo en supermercados

b) Menos de $\$ 10.000$

c) De $\$ 10.000$ a $\$ 20.000$

d) Sobre $\$ 20.000$

2. Del dinero destinado a las compras con la tarjeta de alimentación en supermercado que cantidad semanal de os siguientes productos adquiere:

\begin{tabular}{|c|c|c|c|c|}
\hline Alimento & $\mathrm{a}$ & $b$ & c & $d$ \\
\hline $\begin{array}{ll}\text { 1. } & \text { Bebidas } \\
\text { y jugos } \\
\text { azucarados }\end{array}$ & $\begin{array}{l}<2 \\
\text { litros }\end{array}$ & $\begin{array}{l}2-4 \\
\text { litros }\end{array}$ & $\begin{array}{l}4-6 \\
\text { litros }\end{array}$ & $\begin{array}{l}>6 \\
\text { litros }\end{array}$ \\
\hline $\begin{array}{l}\text { 2. Galletas } \\
\text { azucaradas }\end{array}$ & $<2$ paquetes & 2-4 paquetes & 4-6 paquetes & $>6$ paquetes \\
\hline $\begin{array}{l}\text { 3. Productos de } \\
\text { pastelería }\end{array}$ & $<2$ unidades & 2-4 unidades & 4-6 unidades & $>6$ unidades \\
\hline $\begin{array}{ll}\text { 4. } & \text { Frutas } \\
\text { 5. } & \text { Verduras } \\
\text { 6. } & \text { Pan } \\
\text { 7. } & \text { Embutidos } \\
\text { 8. } & \text { Legumbres } \\
\text { 9. } & \text { Lácteos } \\
\text { 10. } & \text { Cereales }\end{array}$ & $\begin{array}{l}<1 \mathrm{~kg} \\
<1 \mathrm{~kg} \\
<1 \mathrm{~kg} \\
<1 \mathrm{~kg} \\
<500 \mathrm{~g} \\
<2 \text { litros } \\
<1 \mathrm{~kg}\end{array}$ & $\begin{array}{l}1-3 \mathrm{~kg} \\
1-3 \mathrm{~kg} \\
1-3 \mathrm{~kg} \\
1-3 \mathrm{~kg} \\
500 \mathrm{~g}-1 \mathrm{~kg} \\
2-4 \text { litros } \\
1-3 \mathrm{~kg}\end{array}$ & $\begin{array}{l}3-5 \mathrm{~kg} \\
3-5 \mathrm{~kg} \\
3-5 \mathrm{~kg} \\
3-5 \mathrm{~kg} \\
1-3 \mathrm{~kg} \\
4-6 \text { litros } \\
3-5 \mathrm{~kg}\end{array}$ & $\begin{array}{l}>5 \mathrm{~kg} \\
>5 \mathrm{~kg} \\
>5 \mathrm{~kg} \\
>5 \mathrm{~kg} \\
>3 \mathrm{~kg} \\
>6 \text { litros } \\
>5 \mathrm{~kg}\end{array}$ \\
\hline
\end{tabular}

3. Del dinero de la tarjeta de alimentación mensual ¿cuánto utiliza en locales de comida rápida?

a) No la utilizo en locales de comida rápida.

b) Menos de $\$ 10.000$

c) De 10.000 a $\$ 20.000$

d) Sobre $\$ 20.000$

4. Del dinero de la tarjeta de alimentación mensual ¿Cuánto utiliza en menú almuerzo con valor JUNAEB

a) No la utilizo en la compra de menú almuerzo con valor JUNAEB

b) Menos de $\$ 10.000$

c) De $\$ 10.000$ a $\$ 20.000$

d) Sobre $\$ 20.000$

5. Del dinero mensual utilizado en supermercados ¿Cuánto dinero utiliza en frutas y verduras?

a) No compro frutas y verduras

b) Menos de $\$ 5.000$

c) Entre $\$ 5.000$ y $\$ 10.000$

d) Sobre $\$ 10.000$

6. Del dinero mensual utilizado en supermercado ¿Cuánto utiliza en lácteos?

a) No compro lácteos

b) Menos de $\$ 5.000$

c) Entre $\$ 5.000$ y $\$ 10.000$

d) Sobre $\$ 10.000$

7. Del dinero mensual utilizado en supermercado ¿Cuánto utiliza para comprar pan?

a) No compro pan

b) Menos de $\$ 5000$

c) Entre $\$ 5000$ y $\$ 10000$

d) Sobre $\$ 10.000$

8. Del dinero mensual utilizado en supermercado ¿Cuánto utiliza para comprar embutidos?

a) No compro

b) Menos de $\$ 5.000$

c) Entre $\$ 5000$ y $\$ 10.000$

d) Sobre $\$ 10.000$

9. Del dinero mensual utilizado en supermercado ¿Cuánto utiliza para comprar legumbres?

a) No compro legumbres

b) Menos de $\$ 5.000$

c) Entre $\$ 5.000$ y $\$ 10.000$

d) Sobre $\$ 10.000$

10. Cuantos días a la semana consume alimentación en establecimientos de comida rápida.

a) No consumo alimentación en establecimientos de comida rápida.

b) 1 a 3 días

c) 4-5 días

d) 5 a 7 días

11. Cuantos días al mes consume alimentación en establecimientos de comida rápida.

a) No consumo alimentos en establecimientos de comida rápida.

b) 1-7 días

c) 8 - 14 días

d) Sobre 15 días

12. Nombre la preparación de comida que consume con mayor frecuencia en establecimientos de comida rápida. 
participante como muy deficiente, deficiente, regular o adecuada, (tablas 1 y 2 ).

Posteriormente, se realizó una evaluación antropométrica, a través de la medición del peso, talla y circunferencia de cintura, para lo cual se utilizó una balanza digital marca ADE modelo 320600 con capacidad total de $250 \mathrm{~kg}$ y precisión de $50 \mathrm{~g}$, tallímetro portátil marca SECA 213 con rango de medición de 20 - $205 \mathrm{~cm}$ y precisión de $1 \mathrm{~mm}$ y cinta métrica Cescorf con presición de $1 \mathrm{~mm}$. La evaluación nutricional se realizó de manera individual en el Centro de Atención Profesional (CAP) de la Universidad Santo Tomás sede Valdivia. Se clasificó el estado nutricional de cada sujeto de acuerdo al índice de masa corporal (IMC) (5).

Para el análisis estadístico de los datos, se utilizó el programa STATA 12.0 y los test estadísticos Shapiro-Wilk, Bartlett, ANOVA y Correlación de Pearson. Con un poder del $80 \%$, nivel de confianza de 95\%; $p<0.05$.

\section{RESULTADOS}

Los participantes presentaron un IMC promedio de 25.4 $\mathrm{kg} / \mathrm{m}^{2}$ (mínimo $18.4 \mathrm{~kg} / \mathrm{m}^{2}$ y máximo $40,5 \mathrm{~kg} / \mathrm{m}^{2}$ ) que corresponde a sobrepeso. La circunferencia de cintura promedio de los participantes, considerando varones y mujeres, fue 83 $\mathrm{cm}$ (mínimo $65 \mathrm{~cm}$ y máximo $116 \mathrm{~cm}$ ) que corresponde según clasificación a riesgo cardiovascular moderado (tabla 3).

De acuerdo a los resultados de la encuesta JUNATEST, se observó que $80 \%$ de los encuestados utiliza sobre $\$ 10.000$ de la tarjeta de alimentación JUNAEB en supermercados, sin observarse diferencias entre las carreras $(p>0.05)$.
Un $25 \%$ de ellos adquiere con la tarjeta de alimentación más de 6 litros de jugos y bebidas azucaradas al mes, sin observarse diferencias entre carreras ( $p>0.05)$.

Destaca que $38.3 \%$ de los alumnos no utiliza la tarjeta de alimentación para adquirir frutas y verduras. Además sólo $6.7 \%$ adquiere más de $5 \mathrm{~kg}$ de fruta y $16.7 \%$ adquiere más de $5 \mathrm{~kg}$ de verduras al mes con la tarjeta de alimentación JUNAEB, sin detectarse diferencias entre carreras ( $p>0.05)$.

Se presentó una diferencia significativa entre la carrera de psicología y las demás carreras, ya que los alumnos encuestados de la carrera de psicología destinaban una mayor cantidad de dinero de la tarjeta JUNAEB a la adquisición de frutas y verduras $(p<0.05)$, (gráfico 1$)$.

Un $46.7 \%$ de los alumnos no utilizaba la tarjeta para la adquisición de pan y $28.3 \%$ de los participantes adquiere entre 1 y $3 \mathrm{~kg}$ de pan al mes con la tarjeta de alimentación JUNAEB, sin diferencias entre carreras ( $p>0.05)$.

Un $70 \%$ de los alumnos encuestados adquiere menos de $1 \mathrm{~kg}$ de embutidos, 28.3\% entre 1 y $3 \mathrm{~kg}$, y $1.7 \%$ entre 3 y $5 \mathrm{~kg}$ de embutidos al mes con la tarjeta. No se observarón diferencias entre las carreras ( $p>0.05)$.

Cabe destacar que alrededor del $50 \%$ de los encuestados destina hasta $\$ 10.000$ de la tarjeta de alimentación JUNAEB para la adquisición de embutidos; sin diferencias entre las carreras ( $p>0.05)$

Un $63.3 \%$ de los alumnos no utilizaba la tarjeta de alimentación para adquirir legumbres $10 \%$ adquiría entre 0.5 y $1 \mathrm{~kg}$, y $20 \%$ entre 1 y $3 \mathrm{~kg}$ de legumbres al mes con la tarjeta de alimentación, sin diferencias entre las carreras $(p>0.05)$.

TABLA 1

Categorización de calidad de alimentación de acuerdo al puntaje asignado por cada pregunta

Calidad de alimentación

Adecuada

Regular

Deficiente

Muy deficiente
Puntaje asignado por pregunta

4 puntos
3 puntos
2 puntos
1 punto

3 puntos

1 punto

\section{TABLA 2}

Categorización de calidad de alimentación de acuerdo a puntaje total de encuesta JUNATEST

\begin{tabular}{ll}
\hline Calidad de alimentación & Puntaje total de la encuesta \\
\hline Adecuada & $61-80$ puntos \\
Regular & $41-60$ puntos \\
Deficiente & $21-40$ puntos \\
Muy deficiente & $1-20$ puntos \\
\hline
\end{tabular}

TABLA 3

Estado nutricional (IMC) y circunferencia de cintura (CC) promedio según carrera

\begin{tabular}{lccccccc}
\hline Carrera & $\mathrm{N}$ & $\begin{array}{c}\mathrm{IMC}\left(\mathrm{kg} / \mathrm{m}^{2}\right) \\
\text { promedio total }\end{array}$ & $\begin{array}{c}\mathrm{IMC}\left(\mathrm{kg} / \mathrm{m}^{2}\right) \\
\text { mujeres }\end{array}$ & $\begin{array}{c}\mathrm{IMC}\left(\mathrm{kg} / \mathrm{m}^{2}\right) \\
\text { varones }\end{array}$ & $\begin{array}{c}\mathrm{CC}(\mathrm{cm}) \\
\text { promedio total }\end{array}$ & $\begin{array}{c}\mathrm{CC}(\mathrm{cm}) \\
\text { mujeres }\end{array}$ & $\begin{array}{c}\mathrm{CC}(\mathrm{cm}) \\
\text { varones }\end{array}$ \\
\hline Kinesiología & 18 & 24.39 & 24.87 & 20.50 & 82 & 82.56 & 77.75 \\
Enfermería & 29 & 25.71 & 25.74 & 24.84 & 83 & 71.25 & 96.00 \\
Derecho & 7 & 27.59 & 26.65 & 28.29 & 90 & 83.00 & 96.00 \\
Psicología & 6 & 24.98 & 25.66 & 23.63 & 78 & 79.88 & 75.00 \\
\hline
\end{tabular}


Destaca que $95 \%$ de los encuestados destina menos de $\$ 5.000$ en la adquisición de legumbres con la tarjeta. No se observó diferencias entre las carreras ( $p>0.05)$.

Un $11.7 \%$ de los alumnos no utilizaba la tarjeta de alimentación para comprar lácteos mensualmente. Cabe destacar que existe $58,3 \%$ de los participantes que adquiere más de 4 litros de lácteos al mes, destinando entre $\$ 5.000$ y $\$ 10.000$ de la tarjeta de alimentación JUNAEB, sin observarse diferencias entre las carreras ( $p>0.05)$.

Un 26.6\% de los alumnos encuestados adquiere menos de $1 \mathrm{~kg}$ de cereal mensual, $38.3 \%$ entre 1 y $3 \mathrm{~kg}$ y $35 \%$ adquiere sobre $3 \mathrm{~kg}$ de cereales al mes con la tarjeta; sin presentarse diferencias entre las carreras $(p>0.05)$.

Cabe destacar que $73.4 \%$ de los alumnos encuestados utilizaba entre $\$ 0$ y $\$ 20.000$ del dinero de la tarjeta mensual para consumir alimentación en establecimientos de comida rápida. Los alumnos de la carrera de derecho presentaban un consumo menor en establecimientos de comida rápida con la tarjeta de alimentación JUNAEB que los estudiantes de psicología ( $p=0.04)$, (gráfico 2$)$.

Un $43.3 \%$ de los sujetos no utilizaba la tarjeta de alimentación para comprar almuerzos con valor JUNAEB, $45 \%$ utilizaba menos de $\$ 10.000,6.7 \%$ de $\$ 10.000$ a $\$ 20.000$ y $5 \%$ de los estudiantes más de $\$ 20.000$ de la tarjeta de alimentación en adquirir almuerzos con valor JUNAEB.

Es de relevancia que sólo $11.7 \%$ de los alumnos encuestados destina más de $\$ 10.000$ del total de la tarjeta a la compra de almuerzos con valor JUNAEB; sin diferencia significativa entre las carreras ( $p>0.05)$.

Un 20\% de los alumnos no consumía alimentación en establecimientos de comida rápida, sin embargo se destaca que el $80 \%$ restante destina dinero de la tarjeta de alimentación JUNAEB para consumir alimentos en establecimientos de comida rápida durante la semana.

Se observaron diferencias en los estudiantes de la carrera de derecho que consumen alimentación en establecimientos de comida rápida con menor frecuencia semanal que la carrera de psicología ( $p=0.02)$, (gráfico 3 ).

Del total de encuestados; sólo 19\% prefiere consumir comida casera en establecimientos de comida rápida a diferencia del $81 \%$ que prefiere el consumo de alimentos con alto aporte calórico y grasa saturada.

Según el análisis de calidad de alimentación realizado según el uso de la tarjeta JUNAEB la carrera de Kinesiología,

\section{GRÁFICO 1}

Dinero mensual de la tarjeta de alimentación JUNAEB destinado a la adquisición de frutas y verduras.

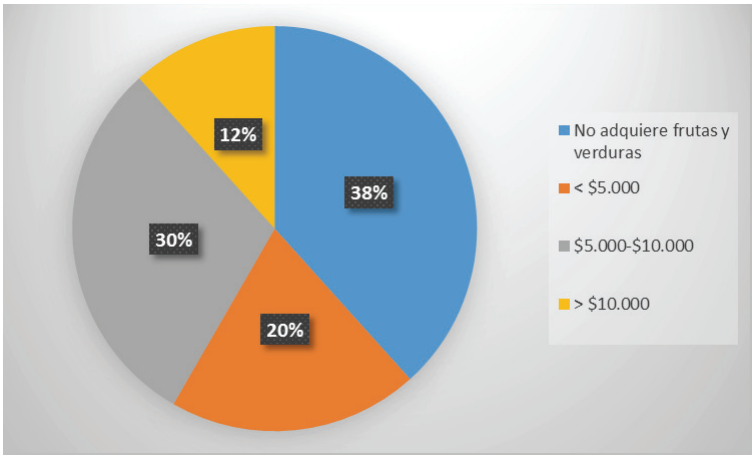

GRÁFICO 2

Dinero mensual de la tarjeta de alimentación JUNAEB destinado a la adquisición de alimentos en establecimientos de comida rápida.

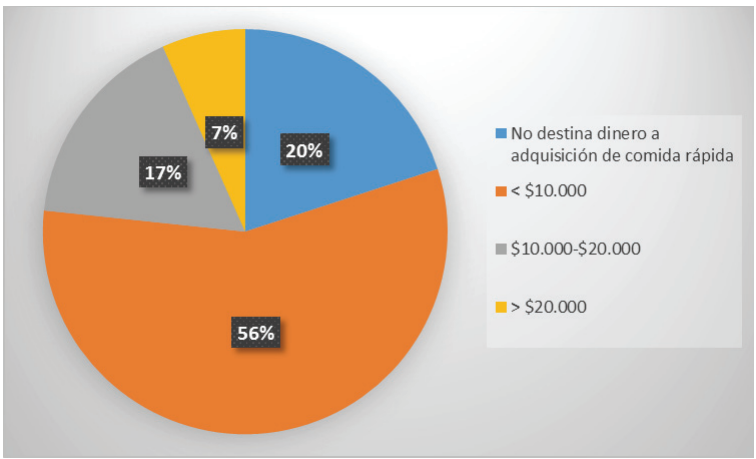


enfermería y derecho mantienen una alimentación regular y sólo la carrera de psicología califica con una alimentación adecuada (tabla 4).

Cabe destacar que la variable estado nutricional en los estudiantes, no presentó relación directa con la calidad de alimentación adquirida con la tarjeta de alimentación JUNAEB, de acuerdo a la categorización del puntaje final de la encuesta JUNATEST (Correlación Pearson 0.08), (tabla 5).

\section{DISCUSION}

La Organización Mundial de la Salud, desde 1980, considera la obesidad como una epidemia global que constituye un problema importante de salud pública (6). En Chile la malnutrición por exceso es una problemática compleja, asociada a la vida moderna en la cual el sedentarismo y los hábitos alimentarios son limitaciones relevantes para una vida saludable (1).

La información sobre alimentación y estilos de vida en el adulto joven es limitada en nuestro país, sin embargo en la última Encuesta Nacional de Salud (2009) se observó que en la población de 15 a 24 años de edad existe 37,8\% de mal nutrición por exceso, esto radica en que la dieta habitual de la población chilena se caracteriza por un alto aporte calórico proveniente de grasas saturadas, colesterol y azúcares simples (1).

Como política pública de ayuda a estudiantes universitarios el Ministerio de Educación otorga una beca de alimentación que tiene por objeto apoyar la permanencia y el egreso de la educación superior a estudiantes vulnerables (4). En Chile no hay evidencia disponible sobre el uso que realizan los universitarios con la tarjeta de alimentación JUNAEB, razón por la cual el objetivo de este estudio fue contribuir a obtener información sobre el uso de la tarjeta de canje y relacionarlo al estado nutricional de los estudiantes universitarios.

Se detectó en el grupo estudio un IMC promedio de $25.4 \mathrm{~kg} / \mathrm{m}^{2}$ que corresponde según clasificación a sobrepeso. Este resultado difiere de otras investigaciones realizadas en estudiantes universitarios españoles que presentaron un IMC promedio normal (7), y otro estudio realizado en nuestro

\section{GRÁFICO 3}

Alimentos de preferencia adquiridos con la tarjeta JUNAEB.

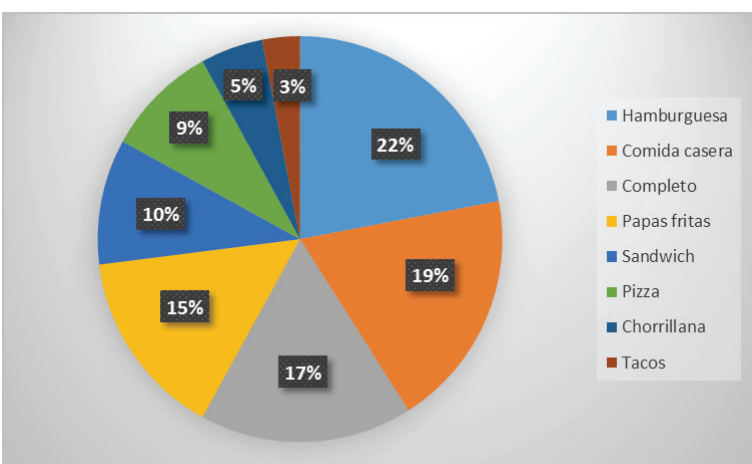

TABLA 4

Diferencias entre las carreras sobre calidad de alimentación según uso de tarjeta JUNAEB

\begin{tabular}{lcccc}
\hline Carrera & $\begin{array}{c}\text { Muy deficiente } \\
1-20 \mathrm{pts}\end{array}$ & $\begin{array}{c}\text { Deficiente } \\
21-40 \mathrm{pts}\end{array}$ & $\begin{array}{c}\text { Regular } \\
41-60 \mathrm{pts}\end{array}$ & $\begin{array}{c}\text { Adecuada } \\
61-80 \mathrm{pts}\end{array}$ \\
\hline Kinesiología & & 58 & \\
Enfermería & & 59 & 63 \\
Derecho & & 60 & 63 \\
Psicología & & & \\
\hline
\end{tabular}

TABLA 5

Relación de estado nutricional según IMC y la calidad de alimentación según puntaje de encuesta JUNATEST

\begin{tabular}{ccc}
\hline & Puntaje JUNATEST & IMC \\
\hline Puntaje JUNATEST & 1.0000 & \\
IMC & 0.0849 & 1.0000 \\
\hline
\end{tabular}

Correlación de Pearson 
país que involucró 11 regiones en la cual la media de IMC se registró en el límite superior de normalidad (8).

Al analizar la encuesta JUNATEST se observó que 93\% de los estudiantes hace uso de la tarjeta en supermercados. De los productos que adquieren, destaca que la cuarta parte de la muestra destina dinero de la tarjeta para la adquisición de más de 6 litros de jugos y bebidas azucaradas mensualmente, no obstante, por reglamento se prohíbe la venta de dichos productos (4). Según el estudio "Chile Saludable" las bebidas azucaradas son las preferidas de los chilenos con un consumo per-cápita de 170 litros anuales entre bebidas gaseosas, jugos y aguas embotelladas (9). Atalah et al, observaron que 35\% de los estudiantes universitarios con tarjeta JUNAEB consumía bebidas gaseosas todos los días, aunque no específicamente con tarjeta (8).

El 28\% de los estudiantes destinaban dinero de la tarjeta JUNAEB en adquirir galletas azucaradas; $85 \%$ de los alumnos no destinaba dinero de la tarjeta para la adquisición de productos de pastelería, resultado que contrasta con los obtenidos por Atalah et al, quienes indicaron que $63 \%$ de los alumnos con tarjeta JUNAEB consumía productos de pastelería todos los días (8).

La dieta de la población chilena se caracteriza por el bajo consumo de frutas y verduras. La última Encuesta Nacional de Salud, indicó que sólo 15,7\% de la población chilena consumía 5 o más porciones de frutas y verduras al día (1). Este estudio refleja resultados similares, ya que $47 \%$ de la muestra adquiere menos de $1 \mathrm{~kg}$ de frutas con tarjeta JUNAEB. Los resultados aumentan para el grupo de verduras, ya que $50 \%$ adquiere menos de $1 \mathrm{~kg}$ al mes. De estos estudiantes 38\% indicó que no adquiere frutas y verduras con la tarjeta de alimentación JUNAEB. Se observó una diferencia significativa, ya que estudiantes de psicología que destinaban mayor cantidad de dinero de la tarjeta de alimentación JUNAEB en la adquisición de frutas y verduras, que las demás carreras encuestadas.

Chile es el segundo país después de Alemania con mayor consumo de pan, con un promedio anual de 98 kilos per cápita $(10,11)$, sin embargo, en los resultados de este estudio $47 \%$ de los estudiantes no destina dinero de la tarjeta de alimentación JUNAEB a la adquisición de pan. No obstante, 53\% restante destina hasta $\$ 10.000$ mensual para la adquisición de este producto.

El Instituto Nacional de Estadísticas (INE) informó del comportamiento de los chilenos en el consumo de carnes y fiambres, destacando que la elaboración de embutidos registró el año 2013 un record de 275.000 toneladas, con un consumo per cápita de 15,6 kg. Los resultados reflejados en la encuesta JUNATEST indican que el 52\% no adquiere embutidos, sin embargo $40 \%$ de los estudiantes que destina hasta $\$ 5.000$ de la tarjeta para la adquisición de estos productos que en su mayoría son vienesas, mientras que el $8 \%$ restante destina poco más de $\$ 5.000$ de la tarjeta a la adquisición de estos productos (12).

Según la Oficina de Estudios y Políticas Agrarias el consumo de legumbres ha disminuido considerablemente en los últimos años. El año 2011 el consumo de leguminosas per cápita de $2,7 \mathrm{~kg}$ anual (10). Los resultados muestran que $63 \%$ de los estudiantes encuestados no adquiere leguminosas con la tarjeta de alimentación y que sólo $32 \%$ destina menos de $\$ 5.000$ para su adquisición. Los resultados coinciden con el consumo de estos productos a nivel nacional (13) y también con el estudio de Atalah et al, quienes concluyeron que alrededor del 30\% de los estudiantes con beca de alimentación
JUNAEB no consumía legumbres (8).

En los últimos 20 años el consumo de leche per cápita en Chile ha registrado un incremento cercano al 25\% (10), lo que refleja el impacto que ha tenido este producto en la población chilena. Sólo $12 \%$ de los estudiantes no adquiere lácteos con la tarjeta de alimentación JUNAEB, mientras que el 83\% destina hasta $\$ 10.000$ del dinero de la tarjeta. En general el consumo de lácteos es más alto que otros grupos de alimentos, así lo demuestra un estudio realizado en estudiantes universitarios de México y Barcelona que coincide con los resultados de este estudio (14).

Los cereales son el producto más adquirido con la tarjeta de alimentación JUNAEB y corresponden a la base de la dieta habitual, en este grupo se incluye arroz, tallarines, avena y cereales de desayuno. Existía 35\% de estudiantes que adquiere más de $3 \mathrm{~kg}$ de cereales al mes y 13\% destina una importante cantidad de dinero de la tarjeta para la adquisición de 5 o más $\mathrm{kg}$ de cereales al mes.

En los últimos años el comercio en establecimientos de comida rápida ha crecido fuertemente (15), así como la oferta y demanda de estos productos que se caracterizan por un elevado aporte calórico, de grasas saturadas y azúcares simples. Se permite el uso de tarjeta de alimentación JUNAEB en estos establecimientos, ya que la mayoría ofrece un menú JUNAEB bajo un costo de $\$ 1.300$, sin embargo los resultados de este estudio indican que del total de encuestados $80 \%$ utiliza la tarjeta de alimentación en estos establecimientos y que $43 \%$ no adquiere el menú JUNAEB ofrecido en estos "patios de comida". Además alrededor del 70\% de los estudiantes consume de 1 a 3 días a la semana alimentación tipo bocadillos en establecimientos de comida rápida. Sólo 19\% de los alumnos que utilizan tarjeta JUNAEB en establecimientos de comida rápida prefiere consumir almuerzo tipo menú, mientras que $81 \%$ de los encuestados prefiere el consumo de productos con alto aporte calórico, destacándose la preferencia de hamburguesas, completo o hot-dog y papas fritas como parte del consumo habitual, (gráfico 4).

Se debe considerar que en Chile la ayuda económica de la beca de alimentación JUNAEB para educación superior (\$32.000) no es suficiente para la adquisición de todos los alimentos que componen la base de una alimentación saludable. Los beneficiados son parte de la población más vulnerable que ingresa a la educación superior en nuestro país y además su uso es destinado a adquirir alimentos para todo el grupo familiar del estudiante incrementando esta problemática. Cabe destacar que en el presente estudio el uso de la tarjeta JUNAEB categorizada de acuerdo a la calidad de alimentación adquirida no presentó relación con el estado nutricional de los participantes de la investigación.

\section{RESUMEN}

Introducción: La dieta habitual chilena se caracteriza por un elevado aporte calórico proveniente de grasas saturadas, colesterol, azúcares y sodio, presentes en alimentos como frituras, productos de panadería y pastelería, embutidos, golosinas y bebidas azucaradas. Esta situación, además del déficit de actividad física poblacional ha contribuido a deteriorar el estado nutricional del adulto joven; reflejada en la encuesta nacional de salud del año 2010 que en jóvenes de 15-24 años identificó $10,9 \%$ de obesidad, $13.2 \%$ de colesterol elevado y $79.3 \%$ de sedentarismo. Objetivo: Relacionar el uso de tarjeta JUNAEB con el estado nutricional de estudiantes de la Universidad Santo Tomás, sede Valdivia durante el segundo semestre del año 2014. Metodología: Estudio de corte trans- 
versal; se aplicó una encuesta "JUNATEST" a 60 estudiantes de la Universidad Santo Tomás con el fin de evaluar la calidad de alimentación que adquieren con el uso de la tarjeta JUNAEB. Posteriormente se relacionó la calidad de alimentación con el estado nutricional de los participantes. Análisis estadístico: Se utilizó el programa STATA 12.0 y los test Shapiro Wilk, Barlett, ANOVA y correlación de Pearson. Para el cálculo del tamaño muestral se utilizó un poder del $80 \%$, un nivel de confianza de $95 \%$, un error de $5 \%$, y un valor $\mathrm{p}<0.05$.

Palabras clave: Tarjeta JUNAEB, alimentación saludable, jóvenes universitarios, estado nutricional, encuesta JUNATEST.

\section{BIBLIOGRAFIA}

1. Gobierno de Chile. Ministerio de Salud. Encuesta Nacional de Salud (ENS) 2009-2010.

2. Gobierno de Chile. Ministerio de Salud. Situación Epidemiológica de las ECNT en Chile, Reporte de vigilancia de Enfermedades Crónicas No Transmisibles (ECNT), 2011.

3. Hábitos alimenticios de los chilenos. Facultad de Ciencias Empresariales, Centro de estudios de opinión ciudadana, Talca. Julio 2006.

4. Junta Nacional de Auxilio Escolar y Becas, JUNAEB. Ministerio de Educación 2014.

5. Organización Mundial de la Salud. Clasificación Índice de Masa Corporal.

6. Acta médica grupo Ángeles. Volumen 8, Nº.4, OctubreDiciembre 2010.

7. Ruiz M E, Valero G T, Ávila T J, et al; Estudio de hábitos alimentarios y estilos de vida de los universitarios españoles. Patrón de consumo de bebidas fermentadas. Fundación Española de la Nutrición. Universidad CEU San Pablo, Madrid. 2012-2013.

8. Ratner G R, Hernández J P, Martel A J, Atalah S E; Calidad de la alimentación y estado nutricional en estudiantes universitarios de 11 regiones de Chile. Rev Med Chil 2012; 140: 1571-9.

9. Chile saludable: oportunidades y desafíos de innovación: Volumen 1. Área alimentos y biotecnología de Fundación Chile y Elige vivir sano, Ministerio de Salud, Chile 2013.

10. Consumo aparente de principales alimentos en Chile. ODEPA, Ministerio de Agricultura. Agosto de 2012.

11. La Industria Latinoamericana del pan en cifras. Federación Chilena de Industriales Panaderos.

12. Instituto Nacional de Estadísticas Chile. Boletín de publicaciones carnes y cecinas, 2013.

13. Universidad de Chile. Facultad de Economía y Negocios, centro de microdatos. Facultad de Medicina, Escuela de Nutrición. Encuesta Nacional e Consumo Alimentario, informe final.

14. Díaz M M, Riba M, Rodríguez G A, Mora M. Patrón alimentario de estudiantes universitarios: Comparación entre culturas. Rev Esp Nutr Comun 2005; 11(1):8-11.

15. Guía para el control y prevención de la contaminación industrial rubro elaboración de comidas rápidas, Comisión Nacional del Medio Ambiente. Diciembre 2000. 